\title{
Phantoms models to characterize influenza hemagglutinin-based vaccines
}

\author{
Dustin M. McCraw ${ }^{1}$, Audray K. Harris ${ }^{1}$ \\ 1. Structural Informatics Unit, Laboratory of Infectious Diseases, National Institute of Allergy \\ and Infectious Diseases, National Institutes of Health, Bethesda, MD, USA.
}

Despite the availability of seasonal vaccines and antiviral medications, influenza virus continues to be a major health concern and pandemic threat due to the continually changing antigenic regions of the major surface glycoprotein, hemagglutinin (HA). Hemagglutinin is the major antigen to which neutralizing antibodies are elicited. Neutralizing epitopes reside in both the head region composed of HA1 globular domains and the stem region composed of HA2 domains. Highly conserved epitopes within the stem region of HA2 are the targets of a number of broadly neutralizing antibodies and hold promise for the design a universal influenza vaccine [1].

Recombinant subunit vaccines are one of the most popular platforms for immunizing the population against potential influenza outbreaks. Subunit vaccines have been previously reported to form glycoprotein complexes [2], but the morphology of the glycoprotein complexes between different vaccines is largely not understood. A working knowledge of glycoprotein complexes morphology would lend insight as to whether important broadly neutralizing epitopes in the stem region of HA are obscured from antigenic interactions - epitopes which are exposed in the native virus.

Computational phantoms can provide virtual models and have previously been used in the analysis of 3-D images [3]. Similarly, here we developed a method for the 2-D classification of HA complexes to assess the morphological structure of HA glycoprotein complexes. Further, we assessed whether these glycoprotein complexes can be characterized in a 2-D system or whether additional parameters are needed.

For imaging, 120 micrographs of glycoprotein complexes stained with uranyl acetate were collected on a Titan Krios at a nominal magnification of 75000x and $300 \mathrm{keV}$ (Figure 1A). Over 160 HA complexes were manually selected for image processing (Figure 1B). The structure of representative glycoprotein complexes were analyzed (Figure $1 \mathrm{C}$, left) to create parameters for phantom molecules (Figure 1C, center) and individual phantom molecules (Figure 1C, right). While the morphology of individual complexes vary, a pentameric starfish morphology was observed to be the most common. Therefore, our phantoms were developed with HA extending from a centroid position. Using these parameters, we developed methods using bSoft, EMAN, EMAN2, and newly-developed Python code to create a library of phantom molecules to represent the population of pentameric glycoprotein complexes (Figure 1D).

When the data set was aligned to the phantom classes, the resulting class averages resembled the template (Figure 1E). As model bias can significantly affect the outcome of class averages, we then separated particles from each class into different data sets. Using reference-free techniques, the classes were again aligned to form new class averages (Figure 1F). However, in this case, the new class averages did not resemble the pentameric complexes. Therefore, while the particles did correctly separate into their respective classes, we expect that the original class average 
morphologies were the result of model-bias and that the class templates provided did not adequately represent the spectrum of HA complexes morphologies. Within the provided constraints, all pentameric morphologies were represented. However, our results indicated that planar pentameric morphological templates are not adequate to represent the possible 3-D nature of the HA complexes within HA-based vaccines. Further analysis using additional 3-D phantoms with different oligomeric states may be useful in analyzing HA vaccines and correlation vaccine efficacy with the types and proportions of phantom templates.

[1] Yassine, H.M. et al. Nat Med, 21 (2015), p. 1065-70.

[2] McCraw, D.M., et al. Clin Vaccine Immunol, 23 (2016), p. 483-95.

[3] Kuybeda, O., et al. J Struct Biol, 181 (2013), p. 116-27.

A

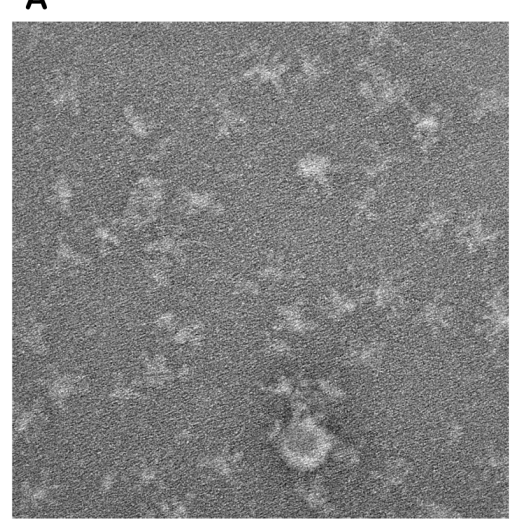

D

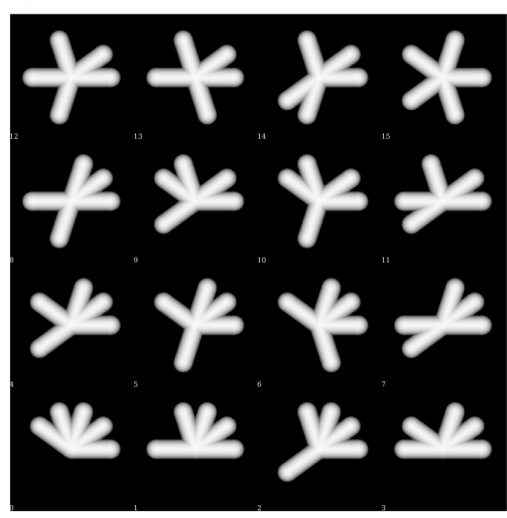

B

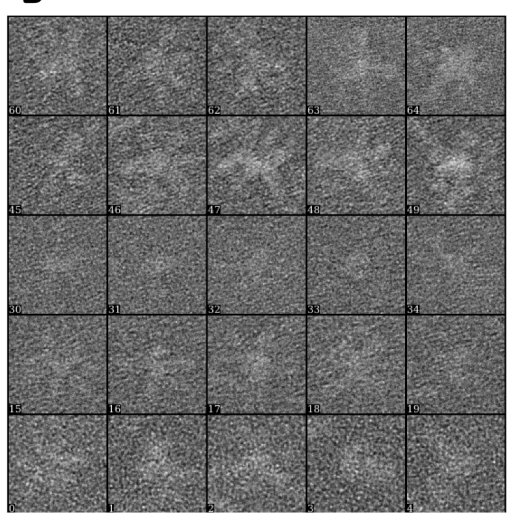

E

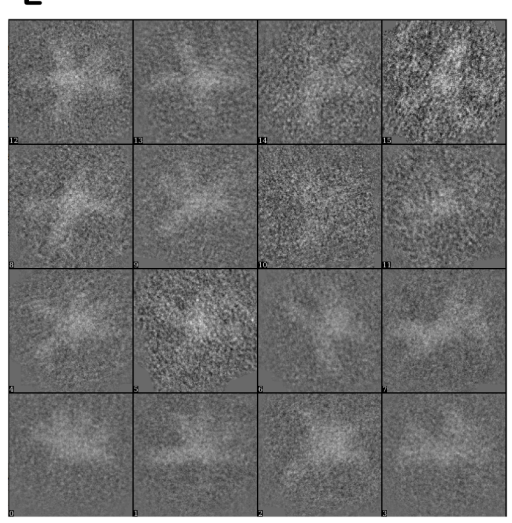

C
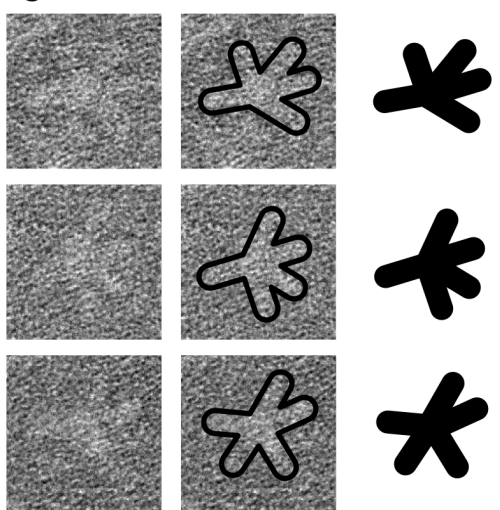

$\mathbf{F}$

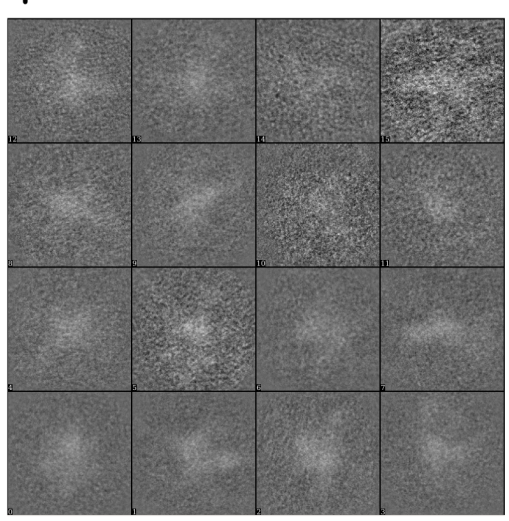

Figure 1. (A) Representative micrograph of HA glycoprotein complexes. (B) Montage of examples of selected individual glycoproteins. (C) Individual complexes (left), outlined (middle) to illustrate previously reported pentameric morphologies (right). (D) Projections of phantom molecules were used to classify out complexes morphologies. (E) Reference-based and (F) reference-free alignment of the particles differ in the extent of observed pentameric morphologies. 